\title{
Regulation of the expression of corticotropin-releasing factor gene by pyroglutamylated RFamide peptide in rat hypothalamic 4B cells
}

\author{
Noriko Ishigame $^{1)}$, Kazunori Kageyama ${ }^{1), 2)}$, Shinobu Takayasu ${ }^{1), 2)}$, Kengo Furumai $^{1)}$, Yuki Nakada ${ }^{1)}$ and \\ Makoto Daimon ${ }^{1)}$ \\ 1) Department of Endocrinology and Metabolism, Hirosaki University Graduate School of Medicine, Hirosaki 036-8562, Japan \\ 2) Department of Endocrinology, Metabolism, and Infectious Diseases, Hirosaki University School of Medicine \& Hospital, \\ Hirosaki 036-8563, Japan
}

\begin{abstract}
Pyroglutamylated RFamide peptide (QRFP), an important regulator of metabolism and energy homeostasis, has orexigenic effects. QRFP acts via a specific receptor, Gpr103. Gpr103 mRNA is expressed in the rat hypothalamic paraventricular nucleus (PVN). In the PVN, corticotropin-releasing factor (CRF), which plays a central role in regulating the stress response and is produced in response to stress, stimulates the release of adrenocorticotropic hormone from the anterior pituitary. We hypothesized that QRFP regulates CRF gene expression directly in the hypothalamus, and thus examined the direct effect of QRFP on the promoter activity and mRNA levels of CRF in hypothalamic cells. To examine these pathways, we used hypothalamic 4B cells, a homologous PVN neuronal cell line. Gpr103a and Gpr103b mRNA, and Gpr103 (a and b) proteins were expressed in the hypothalamic cells. The Gpr103 mRNA and protein levels were increased by QRFP. QRFP also stimulated CRF mRNA levels and CRF promoter activity directly in 4B cells following their transfection with the CRF promoter. The protein kinase A (PKA) and protein kinase C (PKC) pathways were involved in the QRFP-induced increases in CRF promoter activity. QRFP stimulated cAMP response element-binding protein (CREB) phosphorylation. CREB phosphorylation was inhibited by a PKC inhibitor. PKC-dependent signaling would be upstream of the CREB phosphorylation. Thus, QRFP-dependent pathways are involved in the regulation of CRF gene expression in the hypothalamus.
\end{abstract}

Key words: QRFP, Corticotropin-releasing factor, Hypothalamus, Stress

PYROGLUTAMYLATED RFAMIDE PEPTIDES (QRFP), including a 26-aa (QRFP26) and a 43-aa (QRFP43) peptides, are important regulators of metabolism or energy homeostasis. QRFP has orexigenic effects in rodents [1-5]. QRFP also regulates behavioral arousal, blood pressure, locomotor activity, and aldosterone and insulin secretion $[2,6,7]$. QRFP acts via a specific receptor, Gpr103, which belongs to the G proteincoupled receptor superfamily $[2,8]$. QRFP43 exhibits more potent agonistic activity than QRFP26 [9].

Gpr103 mRNA is expressed in the hypothalamus and pituitary of humans and mice [2, 8]. Gpr103

Submitted Apr. 4, 2016; Accepted Jun. 27, 2016 as EJ16-0172 Released online in J-STAGE as advance publication Jul. 23, 2016 Correspondence to: Kazunori Kageyama, M.D., Ph.D., Department of Endocrinology and Metabolism, Hirosaki University Graduate School of Medicine, 5 Zaifu-cho, Hirosaki 036-8562, Japan. E-mail: kkageyama@hkg.odn.ne.jp

(c) The Japan Endocrine Society
mRNA is expressed in the rat hypothalamic paraventricular nucleus (PVN) [10]. There are two murine homologues of human Gpr103, termed Gpr103a and Gpr103b [2]. QRFP binds and activates both Gpr103a and Gpr103b [2]. QRFP is an endogenous ligand for the Gpr103 in pancreatic $\beta$-cells and skeletal muscle cells $[11,12]$.

Corticotropin-releasing factor (CRF), a major regulatory peptide in the hypothalamic-pituitary-adrenal (HPA) axis $[13,14]$, mediates a variety of physiological functions, including regulation of the HPA axis activity during periods of stress [15]. CRF, produced in the PVN in response to stress [16], is secreted into the pituitary portal circulation and triggers the synthesis of adrenocorticotropic hormone (ACTH) via CRF receptor type 1 in the anterior pituitary. ACTH then stimulates glucocorticoid release from the adrenal glands [16]. CRF is a potent anorexic peptide that 
plays an important role in inhibiting the central regulation of feeding behavior [17]. Because the HPA axis is an important regulator of metabolism through the actions of corticosterone, it is of interest to clarify in detail the actions of CRF on the HPA axis.

We hypothesized that QRFP regulates CRF gene expression directly in hypothalamus, and thus examined the direct effect of QRFP on the promoter activity and mRNA levels of CRF in hypothalamic cells. To explore this process, we used a homologous PVN neuronal cell line, hypothalamic 4B, because these cells express CRF, arginine vasopressin, CRF receptor type 1 , and glucocorticoid receptors and show characteristics of the parvocellular neurons of the PVN [18]. We also explored the involvement of protein kinase pathways by using various kinase inhibitors in the $4 \mathrm{~B}$ cells.

\section{Materials and Methods}

\section{Materials}

Rat fetal hypothalamic 4B cells, developed from primary hypothalamic culture, were provided by Dr. John Kasckow [18]. QRFP43 was purchased from the Peptide Institute (Osaka, Japan). H89 was purchased from Seikagaku Corporation (Tokyo, Japan). The protein kinase A (PKA) inhibitor 14-22 amide (PKAi), and protein kinase $\mathrm{C}$ (PKC) inhibitors Ro-32-0432 and bisindolylmaleimide I (BIM) were purchased from Calbiochem (San Diego, CA).

\section{Cell culture}

4B cells were incubated in Dulbecco's modified Eagle's medium (DMEM)/F12 supplemented with $20 \%$ fetal bovine serum, $100 \mu \mathrm{g} / \mathrm{mL}$ streptomycin, and $100 \mathrm{U} / \mathrm{mL}$ penicillin at $37^{\circ} \mathrm{C}$ in a humidified atmosphere of $5 \% \mathrm{CO}_{2}$ and $95 \%$ air. Cells were plated at $5 \times$ $10^{4}$ cells/well for 3 days before each experiment. One day before each experiment, the cells were washed and then serum-starved overnight in DMEM/F12 supplemented with $0.2 \%$ bovine serum albumin (BSA). On the experiment day, cells were incubated in medium with vehicle or QRFP. At the end of each experiment, total cellular RNA or protein was collected and stored at $-80^{\circ} \mathrm{C}$ until the appropriate assay was performed.

\section{RNA extraction}

Total cellular RNA was extracted using an RNeasy Mini Kit (Qiagen, Hilden, Germany) according to the manufacturer's protocol. Then, cDNA was synthe- sized from the total RNA $(0.5 \mu \mathrm{g})$ using random hexamers as primers with the SuperScript First-Strand Synthesis System for reverse transcriptase-polymerase chain reaction (RT-PCR) (Invitrogen Corp., Carlsbad, $\mathrm{CA}$ ), according to the manufacturer's instructions.

\section{Reverse transcriptase-polymerase chain reaction}

RT-PCR was carried out in a programmable thermal controller (Bio-Rad, Hercules, CA) with the following oligonucleotide primers [19]: Gpr103a-forward (F) (5'-GCACGGAAGCCTGGGAAT-3'), Gpr103areverse (R) (5'-ATGTGTCTCCTTTGGTTTCTTCCA -3'), Gpr103b-F (5'-TGTGATGCACAGAAGAGTGA AGTTAG-3'), and Gpr103b-R (5'-TGCTGCCCCCA AATGCT-3'). The cycling conditions for Gpr103a and Gpr103b were $1 \times\left(95^{\circ} \mathrm{C}, 10 \mathrm{~min}\right), 35 \times\left(95^{\circ} \mathrm{C}, 15 \mathrm{~s}\right.$; $\left.60^{\circ} \mathrm{C}, 1 \mathrm{~min} ; 72^{\circ} \mathrm{C}, 1 \mathrm{~min}\right)$, and $1 \times\left(72^{\circ} \mathrm{C}, 5 \mathrm{~min}\right)$. A total RNA sample was used for negative control for RT-PCR. Products were separated by electrophoresis on a $1.2 \%$ and $4.5 \%$ agarose gel containing ethidium bromide for Gpr103a and Gpr103b, respectively. The expected sizes of the PCR products for Gpr103a and Gpr103b were 104 bp and 79 bp, respectively.

\section{Real-time RT-PCR}

Real-time RT-PCR was performed to examine the effect of QRFP $(1 \mathrm{nM}-1 \mu \mathrm{M})$ on changes in mRNA levels. The resulting cDNAs were subjected to realtime RT-PCR as follows. The expression level of rat Gpr103a or Gpr103b was evaluated using quantitative real-time RT-PCR based on the previous mentioned specific sets of primers and following probes: Gpr103a Probe (5'-FAM-AGCAAAGTTATCTCGA CCACAGCGTCCA-TAMRA-3') or Gpr103b Probe (5'-FAM-TGTGGGAGAGAGAATCCTGTAGAG ATCA-TAMRA-3'). The expression level of rat CRF mRNA was evaluated using quantitative realtime RT-PCR based on the following specific sets of primers and probes: CRF-F (5'-TGGATCTCACCT TCCACCTTCTG-3'); CRF-R (5'-CCGATAATCT CCATCAGTTTCCTG- $\left.3^{\prime}\right)$; and Probe (5'-FAMGCCAGGGCAGAGCAGTTAGC-TAMRA-3'). Beta2-microglobulin, which was not changed by any treatment, was used as a housekeeping gene to normalize the expression results (Assays-on-Demand Gene Expression Products; Applied Biosystems, Foster City, CA). Each reaction consisted of $1 \times$ TaqMan Universal PCR Master Mix (Applied Biosystems), $1 \times$ Assayson-Demand Gene Expression Product (Rn00560865 
$\mathrm{m} 1$ for rat $\beta 2$-microglobulin) or a set of CRF primers and probes, and $1 \mu \mathrm{L}$ of cDNA in a total volume of 25 $\mu \mathrm{L}$. The following parameters were used in an ABI PRISM 7000 Sequence Detection System (Applied Biosystems): $95^{\circ} \mathrm{C}$ for $10 \mathrm{~min}, 40$ cycles at $95^{\circ} \mathrm{C}$ for $15 \mathrm{~s}$, and $60^{\circ} \mathrm{C}$ for $1 \mathrm{~min}$. The above assays involved specific sets of primers and a TaqMan probe spanning the exon/exon junction and should not, therefore, have been influenced by DNA contamination. Data were collected and recorded with ABI PRISM 7000 SDS software (Applied Biosystems) and expressed as a function of the threshold cycle $\left(\mathrm{C}_{\mathrm{T}}\right)$. The amplification efficacies for each gene of interest and the housekeeping gene amplimers were found to be identical when analyzed with diluted samples [20].

Relative quantitative gene expression was calculated by the $2^{-\Delta \Delta \mathrm{C}_{\mathrm{T}}}$ method. In brief, for each sample assayed, the $\mathrm{C}_{\mathrm{T}}$ of the reactions amplifying the gene of interest and a reference gene were determined. The $\mathrm{C}_{\mathrm{T}}$ of the gene of interest of each sample was corrected by subtracting the $\mathrm{C}_{\mathrm{T}}$ for the housekeeping gene $\left(\Delta \mathrm{C}_{\mathrm{T}}\right)$. Untreated controls were chosen as reference samples, and the $\Delta \mathrm{C}_{\mathrm{T}}$ for all experimental samples was reduced by the average $\Delta \mathrm{C}_{\mathrm{T}}$ for the control samples $\left(\Delta \Delta \mathrm{C}_{\mathrm{T}}\right)$. Finally, the abundance of the experimental mRNA relative to that of the control mRNA was calculated with use of the formula $2^{-\Delta \Delta \mathrm{C}_{\mathrm{T}}}$.

\section{Western blot analysis}

Western blot analysis was performed to examine the effect of QRFP $(100 \mathrm{nM})$ on changes in the protein expression of Gpr103, phosphorylated cAMP response element-binding protein (pCREB), and CREB [21]. Beta-actin was used as a housekeeping protein. The cells were washed twice with phosphate-buffered saline (PBS) and lysed with Laemmli sample buffer. Cell debris was pelleted by centrifugation and the supernatant was recovered. The samples were boiled and subjected to electrophoresis on a 4-20\% gradient polyacrylamide gel, and proteins were transferred to a polyvinylidene fluoride membrane (Daiichi Kagaku, Tokyo, Japan). After blocking with Detector Block ${ }^{\circledR}$ buffer (Kirkegaard \& Perry Laboratories, Gaithersburg, $\mathrm{MD})$, the membrane was incubated for $1 \mathrm{~h}$ with each antibody (anti-Gpr103 (a and b) antibody [1:1,000 dilution], which detected both Gpr103 ( $\mathrm{a}$ and $\mathrm{b}$ ) proteins (Bioworld Technology Inc., Louis Park, MN); anti-pCREB [1:500 dilution]/CREB [1:1,000 dilution] antibodies (Cell Signaling Technology, Beverly, MA); and anti- $\beta$-actin [1:5,000 dilution] antibody, ab8227, Abcam, Cambridge, MA), washed with PBS containing $0.05 \%$ Tween 20 , and incubated with horseradish peroxidase-labeled anti-rabbit immunoglobulin G (1:20,000 dilution; Daiichi Kagaku). The chemiluminescent substrate SuperSignal West Pico (Pierce Chemical Co., Rockford, IL) was used for detection, and the membrane was exposed to BioMax film (Eastman Kodak Co., Rochester, NY).

\section{Construct and transfection}

Cells were transfected with a CRF promoter-driven luciferase reporter construct to examine the activity of the promoter [21]. A 1,077-base pair (bp) restriction fragment containing the human CRF promoter ( -907 to +170 relative to the proximal transcription start point) was obtained by PCR. The PCR products were then confirmed by sequence analysis. The DNA fragments were used to produce the CRF promoter-driven luciferase reporter construct, CRF-907luc, by a two-step cloning method. First, the DNA fragment was cloned into a pGEM-T Easy vector (Promega Corp., Madison, WI). The vector was then digested with Kpn I and Hind III and subcloned into the Kpn I and Hind III cloning sites of the pA3-Luc plasmid. This CRF promoter includes a functional cAMP-response element, negative glucocorticoid regulatory element, and serum response element [22].

For the luciferase activity assay, cells were plated in 12 -well (22-mm diameter) culture trays at $60 \%$ confluency. The next day, cells were transfected using the X-tremeGENE 9 DNA Transfection Reagent Kit (Roche Diagnostics, Indianapolis, IN) according to the manufacturer's instructions; $3 \mu \mathrm{L}$ X-tremeGENE/ $1 \mu \mathrm{g}$ DNA was used. Each well contained $0.5 \mu \mathrm{g}$ of total DNA. The culture medium was then replaced with DMEM supplemented with $10 \%$ fetal bovine serum. One day before each experiment, cells were washed and starved overnight using DMEM supplemented with $0.2 \%$ BSA.

\section{Luciferase activity}

A luciferase assay was performed to examine the effect of QRFP (1 nM - $100 \mathrm{nM})$ on changes in the activity of each promoter according to the manufacturer's protocol. At the end of each experiment, cells were washed twice with PBS without $\mathrm{Ca}^{2+}$ or $\mathrm{Mg}^{2+}$, harvested with PicaGene lysis buffer (Toyo Inki, Tokyo, Japan), and centrifuged at 9,660 $\mathrm{g}$ for $2 \mathrm{~min}$. 
We used $20 \mu \mathrm{L}$ of each supernatant. The reactions were started by the injection of $100 \mu \mathrm{L}$ of luciferin solution in PicaGene buffer. Light output was measured for $20 \mathrm{~s}$ at room temperature using a luminometer (Berthold Lumat LB9501, Postfach, Germany). Beta-galactosidase activity was used as an internal control. Cells were treated in triplicate in each experiment, and the average of three independent experiments is shown.

\section{Statistical analysis}

Each experiment was performed three times. Samples were provided in triplicate for each group of experiments. Each value is expressed as the mean \pm standard error of the mean (SEM). Statistical analysis was performed with one-way analysis of variance
(ANOVA), followed by Fisher's protected least-significant difference post-hoc test. The level of statistical significance was set at $P<0.05$.

\section{Results}

\section{Expression of Gpr103a in $4 B$ cells}

Expressions of both Gpr103a and Gpr103b mRNA were detected in rat 4B cells and hypothalamus (Fig. 1A). Both levels were significantly increased $24 \mathrm{~h}$ after addition of $100 \mathrm{nM}$ QRFP in 4B cells (Fig. 1A). In order to examine physiological regulation of Gpr103, expression levels of Gpr103 ( $a$ and b) proteins were studied by western blot. We found that Gpr 103 protein levels were increased $24 \mathrm{~h}$ after addition of $100 \mathrm{nM}$ QRFP in 4B cells (Fig. 1B).

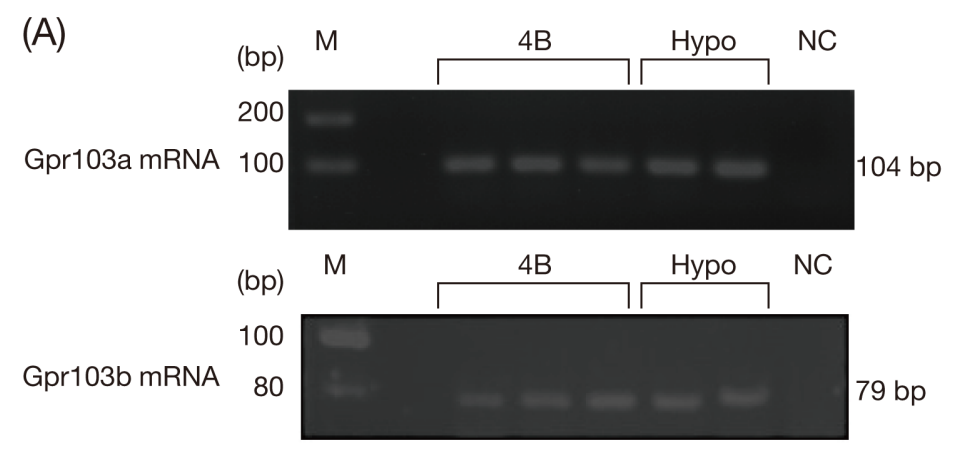

(B)
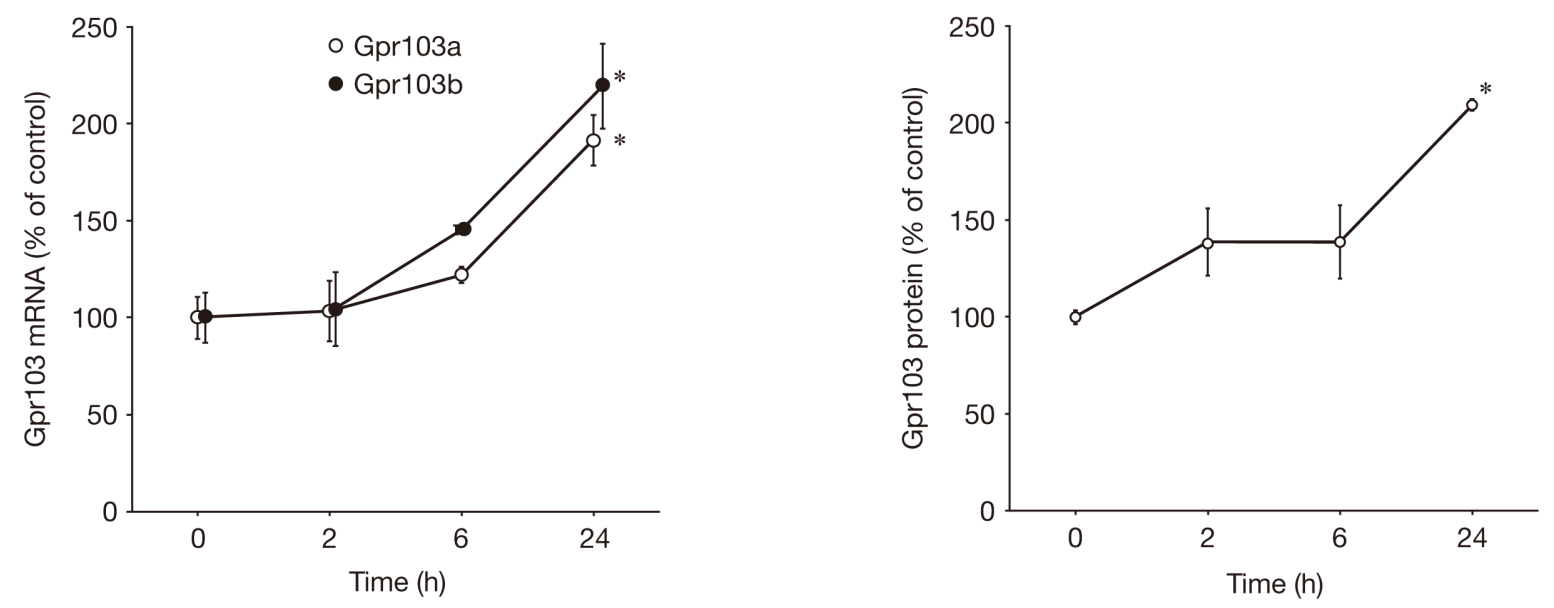

Fig. 1 Expression of Gpr103 mRNA and protein in 4B cells. Cells were treated in triplicate, and the average of three independent experiments and a representative blot are shown. Statistical analysis was performed using one-way ANOVA, followed by post-hoc tests. ${ }^{*} P<0.05$ (compared with control). (A) Effects of QRFP on Gpr103a and Gpr103b mRNA levels in 4B cells. Cells were incubated with medium containing $100 \mathrm{nM}$ QRFP for the durations shown. M, marker; 4B, 4B cells; Hypo, rat hypothalamus (positive control); NC, negative control for RT-PCR. Open circles, Gpr103a; closed circles, Gpr130b. (B) Effects of QRFP on Gpr103 protein levels in 4B cells. Cells were incubated with medium containing $100 \mathrm{nM}$ QRFP for the durations shown. Beta-actin (actin) was used as a housekeeping protein. 


\section{Effects of QRFP on CRF mRNA levels}

We examined the effects of QRFP on the time- and dose-dependent changes in CRF mRNA levels. QRFP significantly stimulated CRF mRNA levels in a dosedependent manner with significant effects observed at $100 \mathrm{nM}$ and $1 \mu \mathrm{M}$ (Fig. 2A). The time-course study showed that QRFP significantly increased CRF mRNA levels (Fig. 2B). The maximum effect of QRFP was observed at $24 \mathrm{~h}$, with an approximately 2.1-fold increase in CRF mRNA levels compared with the basal level.

(A)

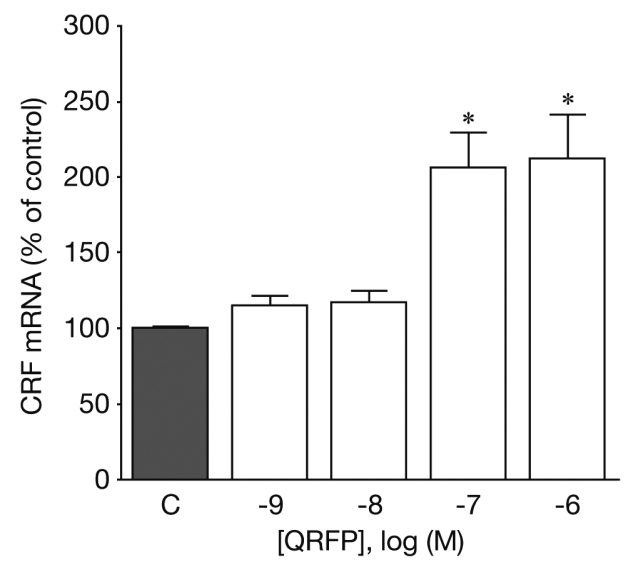

\section{Effects of QRFP on CRF 5'-promoter activity}

We next examined the effects of QRFP on the timeand dose-dependent changes in CRF 5'-promoter activity. QRFP significantly stimulated CRF 5'-promoter activity in a dose-dependent manner with significant effects observed at $100 \mathrm{nM}$ (Fig. 3A). The time-course study showed that QRFP significantly increased CRF 5'-promoter activity (Fig. 3B). The maximum effect of QRFP was observed at $6 \mathrm{~h}$, with an approximately 1.5 fold increase in CRF 5'-promoter activity compared with the basal level.

Fig. 2 Effects of QRFP on CRF mRNA levels in 4B cells. Control cells (C) were treated with medium alone. Cells were treated in triplicate and the average of three independent experiments is shown. Statistical analysis was performed using one-way ANOVA, followed by post-hoc tests. ${ }^{*} P<0.05$ (compared with control). (A) Dose-dependent effects of QRFP on CRF mRNA levels: cells were incubated for $24 \mathrm{~h}$ with medium containing between $1 \mathrm{nM}$ and $1 \mu \mathrm{M}$ QRFP. (B) Time-dependent effects of QRFP on CRF mRNA levels: cells were incubated with medium containing $100 \mathrm{nM}$ QRFP.

(A)

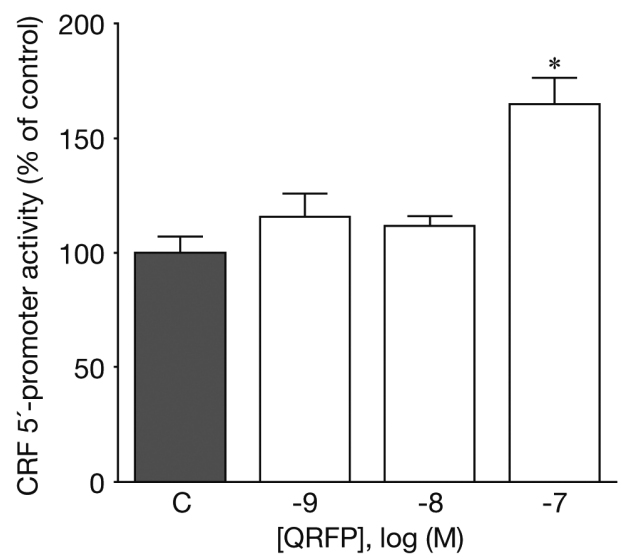

(B)

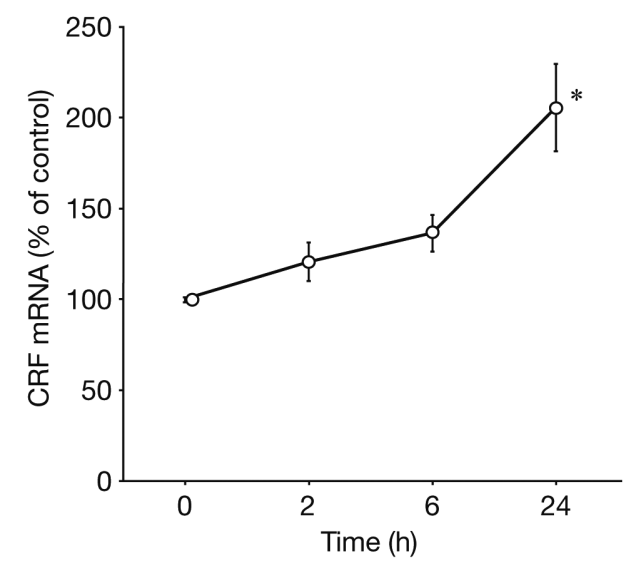




\section{Effects of protein kinase inhibitors on QRFP-induced CRF 5'-promoter activity}

We next investigated which protein kinase pathway was involved in the regulation of QRFP-induced CRF 5 '-promoter activity. To determine whether a protein kinase pathway might be involved in the regulation of CRF 5'-promoter activity, 4B cells were pre-incubated with H89, PKAi, Ro-32-0432, or BIM for $30 \mathrm{~min}$ before incubation with $100 \mathrm{nM}$ QRFP. Incubation of each inhibitor alone did not affect the basal activity of the CRF 5'-promoter for the indicated time.

To determine whether PKA was involved in QRFPinduced CRF 5'-promoter activity, 4B cells were preincubated for 30 min with H89 or PKAi, prior to incubation with QRFP. As shown in Fig. 4, $1 \mu \mathrm{M}$ H89 (Ki for PKA and $\mathrm{PKC}=48 \mathrm{nM}$ and $31.7 \mu \mathrm{M}$, respectively) significantly blocked QRFP-induced CRF promoter activity. PKAi $(100 \mathrm{nM}$; Ki for PKA $=36 \mathrm{nM})$ also significantly blocked QRFP-induced CRF promoter activity. These results suggested that PKA was one of the signaling pathways activated by QRFP.

To determine whether the PKC pathway was involved in QRFP-induced CRF 5'-promoter activity, 4B cells were pre-incubated for $30 \mathrm{~min}$ with Ro-32-0432 or BIM prior to incubation with QRFP. Both Ro-32-0432 (1 $\mu \mathrm{M} ; \mathrm{IC}_{50}$ for $\mathrm{PKC} \beta \mathrm{I}$ and $\mathrm{PKC} \alpha=28$ and $9 \mathrm{nM}$, respectively) and BIM $(1 \mu \mathrm{M}$; Ki for PKC $=10 \mathrm{nM})$, selective PKC inhibitors, significantly inhibited QRFP-induced CRF 5'-promoter activity. Therefore, the PKC pathway was also involved in QRFP-induced signaling.

\section{Effects of QRFP on CREB phosphorylation}

4B cells were incubated with $100 \mathrm{nM}$ QRFP to determine its effects on CREB phosphorylation. A timecourse study showed that CREB phosphorylation was significantly increased $1 \mathrm{~h}$ after addition of $100 \mathrm{nM}$ QRFP in 4B cells (Fig. 5A). Ro-32-0432 (1 $\mu \mathrm{M})$ significantly inhibited QRFP-induced CREB phosphorylation (Fig. 5B).

\section{Discussion}

In the present study, rat hypothalamic 4B cells expressed both Gpr103a and Gpr103b mRNA, and Gpr103 (a and b) proteins. There was no Gpr103 antibody to discriminate between two subtypes for commercial basis. Gpr103b mRNA has been reported to be found in the mouse hypothalamus and the PVN [2], while Gpr103a and Gpr103b mRNA has been found in

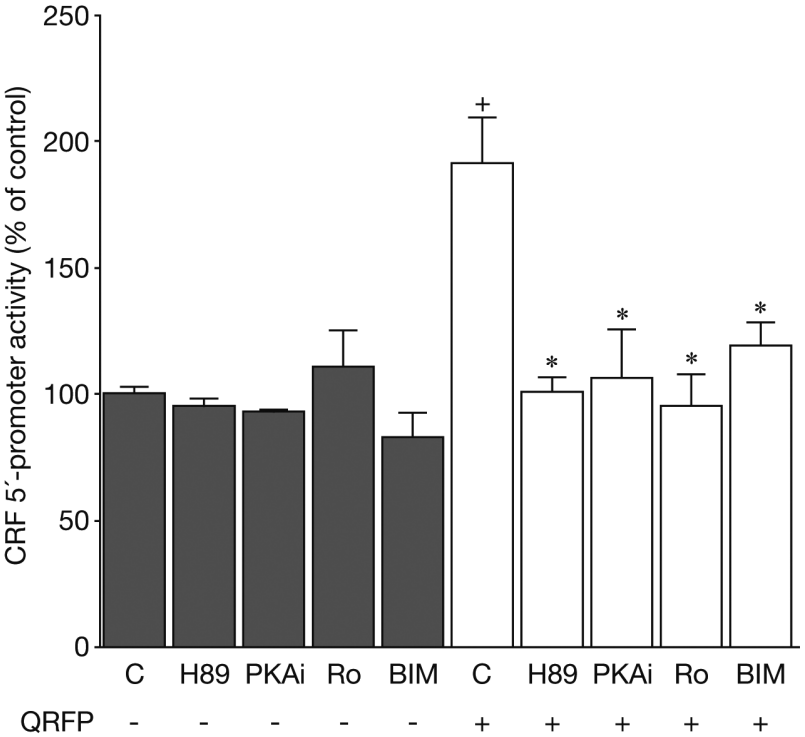

Fig. 4 Effects of protein kinase inhibitors on QRFP-induced CRF 5'-promoter activity in 4B cells. Data are presented as relative activity, and basal luciferase activity without QRFP was set at $100 \%$. Cells were treated in triplicate and the average of three independent experiments is shown. Statistical analyses were performed using oneway ANOVA, followed by post-hoc test. ${ }^{+} P<0.05$ (compared with control). ${ }^{*} P<0.05$ (compared with QRFP alone). Cells transfected with a full-length (CRF907luc) promoter construct were pre-incubated for 30 min with medium containing H89, PKAi, Ro-32-0432 (Ro), BIM, or vehicle, and then incubated for $6 \mathrm{~h}$ with/ without medium containing $100 \mathrm{nM}$ QRFP. Control cells (C) were incubated with the inhibitor vehicle with/ without QRFP.

the rat hypothalamus [19]. These results suggest that QRFP is able to activate CRF neurons via the Gpr103 in rat hypothalamus. Our results also showed that Gpr103 protein levels were directly increased by QRFP. Thus, the results of our study have shown that QRFPdependent pathways are involved in hypothalamic cells. Increases in Gpr103 expression may suggest that QRFP produces a permissive effect on Gpr103.

CRF protein and secretion have failed to be detected in $4 \mathrm{~B}$ cells. Instead, we found that QRFP stimulated the promoter activity and mRNA levels of CRF in hypothalamic 4B cells. The potent PKA inhibitors H89 and PKAi blocked QRFP-induced CRF promoter activity. The potent PKC inhibitors Ro-32-0432 and BIM also inhibited QRFP-induced CRF promoter activity. Therefore, both PKA and PKC pathways were involved in QRFP-induced signaling. QRFP acts on 
(A)
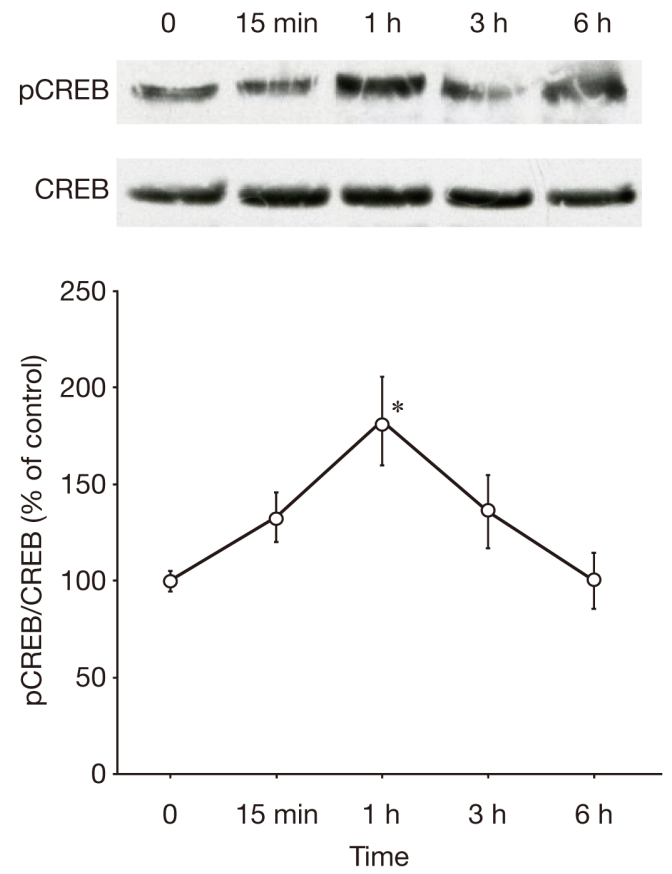

(B)
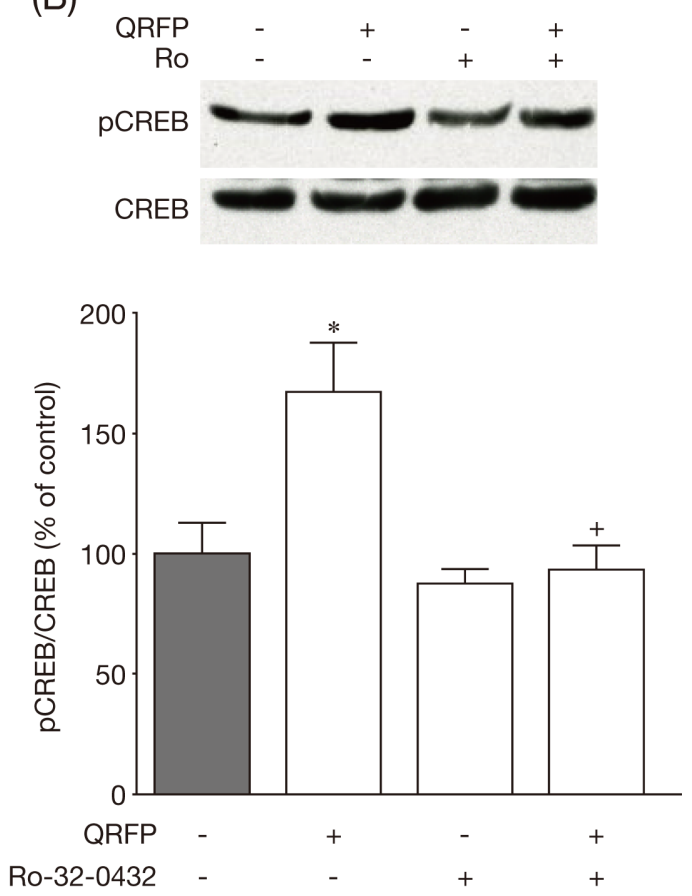

Fig. 5 Effects of QRFP on CREB phosphorylation in 4B cells. Control cells (C) were treated with medium alone. Cells were treated in duplicate and the average of three independent experiments is shown. Statistical analyses were performed using one-way ANOVA, followed by post-hoc test. (A) Time-dependent changes in QRFP-induced CREB phosphorylation in $4 \mathrm{~B}$ cells. ${ }^{*} P<$ 0.05 (compared with control). Cells were incubated with medium containing $100 \mathrm{nM}$ QRFP for the durations shown. Western blot analysis was performed to examine the protein levels of phosphorylated (p) CREB/CREB, and a representative blot is shown. (B) Effects of Ro-32-0432 on QRFP-induced CREB phosphorylation in 4B cells. ${ }^{*} P<0.05$ (compared with control). ${ }^{+} P<0.05$ (compared with QRFP). Cells were pre-incubated for $30 \mathrm{~min}$ with medium containing Ro or vehicle, and then incubated for $1 \mathrm{~h}$ with medium containing $100 \mathrm{nM}$ QRFP or vehicle.

Gpr103 which is Gaq protein-coupled receptor, resulting in increase in intracellular $\mathrm{Ca}^{2+}$ and PKC activity [2]. QRFP can also stimulate CRF gene expression through PKA-related signaling pathways. In fact, in the present study, QRFP stimulated CREB phosphorylation. This CREB phosphorylation was inhibited by a PKC inhibitor. Therefore, PKC-dependent signaling would be upstream of the CREB phosphorylation. Activation of the PKA pathway, which phosphorylates CREB, acts on the CRF promoter [23-25]. A functional cAMPresponse element in the $5^{\prime}$ promoter region is involved in regulating CRF gene expression [21, 23, 24].

Intracerebroventricular injection of QRFP stimulates food intake and general locomotor activity in mice in vivo [2]. QRFP is an orexigenic hormone, whereas CRF would be an anorexigenic or anxiogenic factor in the hypothalamus. QRFP-stimulated $\mathrm{CRF}$ in the hypothalamus would cause ACTH and glucocorticoids secretion. However, it is unclear whether endogenous QRFP stimulates the HPA axis in vivo. Both Gpr103 and QRFP are primarily localized in human and rat adrenal cortex [7,9]. QRFP stimulates cortisol secretion as well as induction of key steroidogenic enzymes in human adrenocortical cells [7]. Glucocorticoids may suppress hypothalamic CRF. On the other hand, it is possible that QRFP stimulates the HPA axis in vivo, and the release of glucocorticoids, representative orexigenic hormone, which helps to increase appetite. In fact, glucocorticoid-induced hyperphagia is observed in CRF over-expressing transgenic mice [26]. Further study is required to determine the effects of QRFP-induced CRF regulation on stress in vivo.

In conclusion, the results of the present study show that Gpr 103 mRNA and protein are expressed in hypothalamic cells. QRFP increases Gpr103 mRNA and protein levels, and stimulates CRF mRNA levels and $\mathrm{CRF}$ promoter activity in $4 \mathrm{~B}$ cells transfected with 
the CRF promoter. The PKA and PKC pathways are involved in the QRFP-induced increases in CRF and promoter activity. QRFP stimulates CREB phosphorylation. CREB phosphorylation is inhibited by a PKC inhibitor. PKC-dependent signaling would be upstream of the CREB phosphorylation. QRFP-dependent pathways appear, therefore, to be involved in the regulation of CRF gene expression in hypothalamic cells. Further studies are required to determine whether this situation also applies in vivo.

\section{Conflict of Interests}

None of the authors have any potential conflicts of interest.

\section{Acknowledgments}

This work was supported by JSPS KAKENHI Grant Number 15K09419.

We thank K Niioka for her technical assistant.

\section{References}

1. Chartrel N, Dujardin C, Anouar Y, Leprince J, Decker A, et al. (2003) Identification of 26RFa, a hypothalamic neuropeptide of the RFamide peptide family with orexigenic activity. Proc Natl Acad Sci US A 100: 15247-15252.

2. Takayasu S, Sakurai T, Iwasaki S, Teranishi H, Yamanaka A, et al. (2006) A neuropeptide ligand of the G protein-coupled receptor GPR103 regulates feeding, behavioral arousal, and blood pressure in mice. Proc Natl Acad Sci U S A 103: 7438-7443.

3. Primeaux SD, Blackmon C, Barnes MJ, Braymer HD, Bray GA (2008) Central administration of the RFamide peptides, QRFP-26 and QRFP-43, increases high fat food intake in rats. Peptides 29: 1994-2000.

4. Zagorácz $\mathrm{O}$, Kovács A, László K, Ollmann T, Péczely L, et al. (2015) Effects of direct QRFP-26 administration into the medial hypothalamic area on food intake in rats. Brain Res Bull 118: 58-64.

5. Schreiber AL, Arceneaux KP $3^{\text {rd }}$, Malbrue RA, Mouton AJ, Chen CS, et al. (2016) The effects of high fat diet and estradiol on hypothalamic prepro-QRFP mRNA expression in female rats. Neuropeptides 58: 103-109.

6. Egido EM, Hernández R, Leprince J, Chartrel N, Vaudry H, et al. (2007) 26RFa, a novel orexigenic neuropeptide, inhibits insulin secretion in the rat pancreas. Peptides 28: 725-730.

7. Ramanjaneya M, Karteris E, Chen J, Rucinski M, Ziolkowska A, et al. (2013) QRFP induces aldosterone production via PKC and T-type calcium channel-mediated pathways in human adrenocortical cells: evidence for a novel role of GPR103. Am J Physiol Endocrinol Metab 305: E1049-1058.

8. Lee DK, Nguyen T, Lynch KR, Cheng R, Vanti WB, et al. (2001) Discovery and mapping of ten novel G protein-coupled receptor genes. Gene 275: 83-91.

9. Fukusumi S, Yoshida H, Fujii R, Maruyama M, Komatsu H, et al. (2003) A new peptidic ligand and its receptor regulating adrenal function in rats. $J$ Biol Chem 278: 46387-46395.
10. Bruzzone F, Lectez B, Alexandre D, Jégou S, Mounien L, et al. (2007) Distribution of 26RFa binding sites and GPR103 mRNA in the central nervous system of the rat. J Comp Neurol 503: 573-591.

11. Allerton TD, Primeaux SD (2015) QRFP-26 enhances insulin's effects on glucose uptake in rat skeletal muscle cells. Peptides 69: 77-79.

12. Granata R, Settanni F, Trovato L, Gallo D, Gesmundo I, et al. (2014) RFamide peptides 43RFa and 26RFa both promote survival of pancreatic $\beta$-cells and human pancreatic islets but exert opposite effects on insulin secretion. Diabetes 63: 2380-2393.

13. Vale W, Spiess J, Rivier C, Rivier J (1981) Characterization of a 41-residue ovine hypothalamic peptide that stimulates secretion of corticotropin and beta-endorphin. Science 213: 1394-1397.

14. Vale W, Vaughan J, Perrin MH (1997) Corticotropinreleasing factor (CRF) family ligands and their receptors. Endocrinologist 7: 3S-9S.

15. Denver RJ (2009) Structural and functional evolution of vertebrate neuroendocrine stress systems. Ann N Y Acad Sci 1163: 1-16.

16. Whitnall MH (1993) Regulation of the hypothalamic corticotropin-releasing hormone neurosecretory system. Prog Neurobiol 40: 573-629.

17. Kawashima S, Sakihara S, Kageyama K, Nigawara T, Suda, T (2008) Corticotropin-releasing factor (CRF) is involved in the acute anorexic effect of a-melanocyte-stimulating hormone: A study using CRF-deficient mice. Peptides 29: 2169-2174.

18. Kasckow J, Mulchahey JJ, Aguilera G, Pisarska M, Nikodemova M, et al. (2003) Corticotropin-releasing hormone $(\mathrm{CRH})$ expression and protein kinase A mediated $\mathrm{CRH}$ receptor signalling in an immortalized hypothalamic cell line. J Neuroendocrinol 15: 521-529.

19. Zhang Q, Qiu P, Arreaza MG, Simon JS, Golovko A, et al. (2007) P518/Qrfp sequence polymorphisms in SAMP6 osteopenic mouse. Genomics 90: 629-635.

20. Kageyama K, Akimoto K, Yamagata S, Sugiyama A, 
Murasawa S, et al. (2012) Dexamethasone stimulates the expression of ghrelin and its receptor in rat hypothalamic 4B cells. Regul Pept 174: 12-17.

21. Kageyama K, Akimoto K, Suda T (2010) Corticotropinreleasing factor gene transcription is directly activated after deprivation of glucocorticoids in hypothalamic cells. J Neuroendocrinol 22: 971-978.

22. Kageyama K, Hanada K, Takayasu S, Iwasaki Y, Sakihara $\mathrm{S}$, et al. (2008) Involvement of regulatory elements on corticotropin-releasing factor gene promoter in hypothalamic 4B cells. J Endocrinol Invest 31: 1079-1085.

23. Seasholtz AF, Thompson RC, Douglass JO (1988) Identification of a cyclic adenosine monophosphateresponsive element in the rat corticotropin-releasing hormone gene. Mol Endocrinol 2: 1311-1319.
24. Spengler D, Rupprecht R, Van LP, Holsboer F (1992) Identification and characterization of a $3^{\prime}, 5^{\prime}$-cyclic adenosine monophosphate-responsive element in the human corticotropin-releasing hormone gene promoter. Mol Endocrinol 6: 1931-1941.

25. Itoi K, Horiba N, Tozawa F, Sakai Y, Sakai K, et al. (1996) Major role of 3',5'-cyclic adenosine monophosphate-dependent protein kinase A pathway in corticotropin-releasing factor gene expression in the rat hypothalamus in vivo. Endocrinology 137: 2389-2396.

26. Nakayama S, Nishiyama M, Iwasaki Y, Shinahara M, Okada Y, et al. (2011) Corticotropin-releasing hormone $(\mathrm{CRH})$ transgenic mice display hyperphagia with increased Agouti-related protein mRNA in the hypothalamic arcuate nucleus. Endocr J 58: 279-286. 